\title{
Chordal and sequentially Cohen-Macaulay clutters
}

\author{
Russ Woodroofe \\ Department of Mathematics \\ Washington University in St. Louis \\ St. Louis, MO, 63130, U.S.A. \\ russw@math.wustl .edu
}

Submitted: May 25, 2011; Accepted: Oct 10, 2011; Published: Oct 24, 2011

Mathematics Subject Classifications: 05E45, 13F55, 13C14, 05C65

\begin{abstract}
We extend the definition of chordal from graphs to clutters. The resulting family generalizes both chordal graphs and matroids, and obeys many of the same algebraic and geometric properties. Specifically, the independence complex of a chordal clutter is shellable, hence sequentially Cohen-Macaulay; and the circuit ideal of a certain complement to such a clutter has a linear resolution. Minimal non-chordal clutters are also closely related to obstructions to shellability, and we give some general families of such obstructions, together with a classification by computation of all obstructions to shellability on 6 vertices.
\end{abstract}

\section{Introduction}

A clutter $\mathcal{C}$ is a hypergraph such that no edge of $\mathcal{C}$ is properly contained in any other edge. For example, any graph is a clutter, as is any $d$-uniform hypergraph. There is a dual relationship between simplicial complexes and clutters, as follows: Given any clutter $\mathcal{C}$, there is an independence complex $I(\mathcal{C})$ which has faces consisting of all subsets of $V(\mathcal{C})$ containing no edge of $\mathcal{C}$. Given any simplicial complex $\Delta$, there is a non-face clutter $\mathcal{C}(\Delta)$ on the same vertex set with edges consisting of the minimal subsets of $V(\Delta)$ which are not faces. Clearly $I(\mathcal{C}(\Delta))=\Delta$ and $\mathcal{C}(I(\mathcal{C}))=\mathcal{C}$.

The non-face clutter of $\Delta$ is perhaps most familiar via the Stanley-Reisner ring of $\Delta$ :

$$
R[\Delta] \triangleq R\left[x_{1}, \ldots, x_{n}\right] /\left(x_{i_{1}} x_{i_{2}} \cdots x_{i_{k}}:\left\{x_{i_{1}}, \ldots, x_{i_{k}}\right\} \text { an edge of } \mathcal{C}(\Delta)\right),
$$

where $V(\Delta)=\left\{x_{1}, \ldots, x_{n}\right\}$. The ideal in this construction is known as the edge ideal or circuit ideal of $\mathcal{C}(\Delta)$.

Recently, a number of papers $[9,12,16,19,30,32]$ have asked what one can say about the algebraic and topological combinatorics of $\Delta$ from the structure of $\mathcal{C}=\mathcal{C}(\Delta)$. A 
particularly successful case has been that where $\mathcal{C}=G$ is a chordal graph. In this case, the independence complex $I(G)$ is vertex decomposable [9, 32], hence shellable [30] and sequentially Cohen-Macaulay [16], while the edge ideal of the complement $\bar{G}$ has a linear resolution [17]. Moreover, the chordal graphs are closely related to the largest family of graphs having independence complexes such that every induced subcomplex is shellable [32]; and the complements of chordal graphs are exactly the graphs with edge ideal having a linear resolution [17].

In the current paper, our purpose is to introduce a family of clutters, which we call chordal clutters, which satisfy similar properties. Chordal clutters generalize several previously studied families, including chordal graphs, circuit clutters of matroids, and acyclic clutters. We will prove:

Theorem 1.1. If $\mathcal{C}$ is a chordal clutter then the independence complex $I(\mathcal{C})$ is shellable and hence sequentially Cohen-Macaulay.

In particular, we obtain a uniform proof of shellability for independence complexes of both chordal graphs and matroids.

We also prove:

Theorem 1.2. Let $\mathcal{C}$ be a d-uniform chordal clutter. Then the circuit ideal of the complement $d$-uniform clutter of $\mathcal{C}$ has a linear resolution.

As previously mentioned, there is a converse to Theorem 1.2 and an interesting partial converse to Theorem 1.1 in the case where $\mathcal{C}$ is a graph. We discuss the possibility of finding such converse results for general chordal clutters. We relate Theorem 1.1 to obstructions to shellability via both examples and computational results, but conclude that non-trivial description of circuit ideals having linear resolution or linear quotients (i.e., a converse to Theorem 1.2 ) is unlikely.

The structure of the papers is as follows. In Section 2 we present the background material. In Section 3 we define $k$-decomposability for non-pure simplicial complexes, and extend many theorems proved by Provan and Billera [27] for pure complexes. In Section 4 we define simplicial vertices in clutters, which naturally leads us to define chordal clutters. We give several examples, including chordal graphs and the circuit clutters of matroids. In Section 5 we prove that the independence complex of any chordal clutter is shellable, as a special case of a more general result about shellability of independence complexes. In Section 6 we recall the basic facts about linear resolutions, combinatorial Alexander duality, and their relationship. We then use the Alexander dual to prove that the circuit ideal of a certain uniform complement of a chordal clutter has a linear resolution, and indeed has linear quotients. We close in Section 7 by relating forbidden minors to chordality with obstructions to shellability. We give several infinite families of these forbidden minors, and characterize by computation with GAP [18] all obstructions to shellability on 6 or fewer vertices that contain no non-shellable link. 


\subsection{Notation}

We will use letters $\mathcal{C}$ and $\mathcal{D}$ for clutters, except in the special case where the clutter is a graph, when we defer to convention and use $G$. Other calligraphic letters such as $\mathcal{F}$ will denote families of objects. Vertices in clutters will be denoted by $v, w$, etc; while circuits (edges) will be denoted with the letter $e$. We use upper case Greek letters such as $\Delta$ and $\Sigma$ for simplicial complexes. Inside a simplicial complex, we use letters $v$ and $w$ for vertices, and lower case Greek letters such as $\sigma$ and $\tau$ for faces.

\section{Acknowledgements}

Eric Emtander explained several aspects of his (alternative) definition of chordal clutter. Volkmar Welker pointed out the connection between $c$-obstructions and Buchsbaum complexes. I have benefited greatly from the interest and advice of John Shareshian.

\section{Background}

\subsection{Simplicial complexes and clutters}

An abstract simplicial complex $\Delta$ on a vertex set $V$ is a set of subsets of $V$ (called faces of $\Delta$ ) such that each subset of a face of $\Delta$ is itself a face of $\Delta$. We do not require that singleton subsets (i.e. elements) of $V$ are faces of $\Delta$. A maximal face is called a facet, and a $d$-dimensional face (having cardinality $d+1$ ) is called a $d$-face.

The join of two simplicial complexes $\Delta_{1}$ and $\Delta_{2}$ on disjoint vertex sets $V_{1}$ and $V_{2}$ is a complex $\Delta_{1} * \Delta_{2}$ on vertex set $V_{1} \cup V_{2}$ with faces $\left\{\sigma_{1} \cup \sigma_{2}: \sigma_{i}\right.$ a face of $\left.\Delta_{i}\right\}$.

A clutter $\mathcal{C}$ on a vertex set $V$ is a set of subsets of $V$ (called circuits or edges of $\mathcal{C}$ ) such that if $e_{1}$ and $e_{2}$ are distinct circuits of $\mathcal{C}$ then $e_{1} \not \subset e_{2}$. Clutters have also been referred to in the literature as Sperner families, or as antichains of sets. To avoid confusion with 1-faces of a simplicial complex, we will usually prefer the term "circuit" over "edge". A $d$ circuit is a circuit consisting of exactly $d$ vertices, and a clutter is d-uniform if every circuit has exactly $d$ vertices. The maximum circuit cardinality of $\mathcal{C}$ is the largest cardinality of any circuit in $\mathcal{C}$, and similarly for the minimum circuit cardinality.

An independent set of $\mathcal{C}$ is a subset of $V$ containing no circuit. Clutters and simplicial complexes are linked via the independence complex $I(\mathcal{C})=\{\sigma \subseteq V: \sigma$ is an independent set of $\mathcal{C}$, and via the clutter of minimal non-faces $\mathcal{C}(\Delta)$. As previously mentioned we have $\mathcal{C}(I(\mathcal{C}))=\mathcal{C}$ and $I(\mathcal{C}(\Delta))=\Delta$.

There are two degenerate simplicial complexes on $V$ : the simplicial complex \{\} with no faces, and the simplicial complex $\{\emptyset\}$ with only the empty set as a face. Notice that $\mathcal{C}(\{\})=\{\emptyset\}$, while $\mathcal{C}(\{\emptyset\})=\{\{v\}: v \in V\}$.

Nondegenerate simplicial complexes admit a geometric realization, a geometric simplicial complex with the same face incidences. When we use geometric or topological terms such as dimension or homotopy type to discuss simplicial complexes, we are referring to the geometric realization.

All clutters and simplicial complexes considered are finite. 


\subsection{Deletions and contractions, links}

Given a simplicial complex $\Delta$, two kinds of subcomplex are of particular interest. If $\sigma$ is a face of $\Delta$, then $\Delta \backslash \sigma$ is obtained from $\Delta$ by removing all faces that contain $\sigma$ from the set system. The star of $\sigma$ is $\operatorname{star}_{\Delta} \sigma=\{$ faces containing $\sigma\}$, and the link of $\sigma$ is the simplicial complex on vertex set $V(\Delta) \backslash \sigma$ with faces

$$
\operatorname{link}_{\Delta} \sigma=\{\tau: \sigma \cap \tau=\emptyset, \sigma \cup \tau \text { is a face of } \Delta\},
$$

Thus $\operatorname{link}_{\Delta} \sigma$ is $\operatorname{star}_{\Delta} \sigma$ with all vertices of $\sigma$ deleted, while $\operatorname{star}_{\Delta} \sigma=\left(\operatorname{link}_{\Delta} \sigma\right) * \sigma$.

Given a clutter $\mathcal{C}$, there are two ways of removing a vertex that are of interest. Let $v \in V(\mathcal{C})$. The deletion $\mathcal{C} \backslash v$ is the clutter on the vertex set $V(\mathcal{C}) \backslash\{v\}$ with circuits $\{e: e$ a circuit of $\mathcal{C}$ with $v \notin e\}$. The contraction $\mathcal{C} / v$ is the clutter on the vertex set $V(\mathcal{C}) \backslash\{v\}$ with circuits the minimal sets of $\{e \backslash\{v\}: e$ a circuit of $\mathcal{C}\}$. Thus, $\mathcal{C} \backslash v$ deletes all circuits containing $v$, while $\mathcal{C} / v$ removes $v$ from each circuit containing it (and then removes any redundant circuits).

A clutter $\mathcal{D}$ obtained from $\mathcal{C}$ by repeated deletion and/or contraction is called a minor of $\mathcal{C}$. If $\mathcal{D}$ is obtained only by deletions we call it an induced subclutter on vertex set $V(\mathcal{D})$. If $\mathcal{D}$ is obtained only by contractions we call it a contraction of $\mathcal{C}$. Notice that if all the vertices contained in a circuit are contracted, then what remains is the clutter $\{\emptyset\}$. It is straightforward to prove that if $v \neq w$ are vertices then $(\mathcal{C} \backslash v) \backslash w=(\mathcal{C} \backslash w) \backslash v$, $(\mathcal{C} / v) / w=(\mathcal{C} / w) / v$, and $(\mathcal{C} \backslash v) / w=(\mathcal{C} / w) \backslash v$.

Example 2.1. A clutter $\mathcal{C}$ is a matroid circuit clutter if it satisfies the weak circuit exchange property: that if $e_{1}$ and $e_{2}$ are circuits of $\mathcal{C}$ containing a common vertex $v$, then there is some circuit $e_{3}$ such that $e_{3} \subseteq\left(e_{1} \cup e_{2}\right) \backslash\{v\}$. In this case the deletion and contraction operations $\mathcal{C} \backslash v$ and $\mathcal{C} / v$ are the usual deletion and contraction in a matroid without loops or coloops. See [26] for additional background and other definitions of matroids. Duval [10] also has a discussion of the relationship between minors in matroids and clutters/simplicial complexes.

We collect the relationships between simplicial complex operations and clutter operations in the following lemma.

Lemma 2.2. Let $\mathcal{C}, \mathcal{D}$ be clutters, and let $v$ be a vertex of $V(\mathcal{C})$. Then

1. $I(\mathcal{C} / v)=\operatorname{link}_{I(\mathcal{C})} v$.

2. $I(\mathcal{C} \backslash v)=I(\mathcal{C}) \backslash v$, considered as a simplicial complex on $V(\mathcal{C}) \backslash v$.

3. If $\mathcal{C}^{\prime}$ consists of the minimal sets of $\mathcal{C} \cup\{\sigma\}$ (where $\sigma$ is an independent set), then $I\left(\mathcal{C}^{\prime}\right)=I(\mathcal{C}) \backslash \sigma$.

4. $I(\mathcal{C} \cup \dot{\mathcal{D}})=I(\mathcal{C}) * I(\mathcal{D})$.

There are obvious dual versions of these which relate a simplicial complex $\Delta$ with its minimal non-face clutter $\mathcal{C}(\Delta)$. 
Example 2.3. If $G$ is a graph (i.e., a clutter with every circuit having cardinality 2), then let $N[v]=\{v$ and all its neighbors $\}$. Then $G \backslash v$ is the usual induced subgraph, while $G / v$ is the induced subgraph $G \backslash N[v]$ together with a singleton circuit for each neighbor $w$ to $v$. In particular, $I(G / v)=I(G \backslash N[v])$.

Remark 2.4. Contraction in a graph $G$ as a clutter should not be confused with contraction in the circuit matroid of $G$ !

Remark 2.5. In a graph, every contraction operation can be expressed (up to singleton circuits) as repeated deletion operations, deleting each vertex in $N[v]$. This does not hold true in general clutters. For example, if $\mathcal{C}$ consists of a single circuit of cardinality 3 , then deleting any vertex leaves a clutter with no circuits, while contracting any vertex leaves a circuit of cardinality 2 .

\subsection{Shellable and Cohen-Macaulay complexes}

We recommend the unfamiliar reader to [2, 3] for background on shellability, and to $[4,5,29]$ for Cohen-Macaulay and sequentially Cohen-Macaulay simplicial complexes, but give a brief review here.

Let $k$ be any field or the ring of integers. A simplicial complex is Cohen-Macaulay if, for every face $\sigma$ (including $\sigma=\emptyset$ ), we have $\tilde{H}_{i}\left(\operatorname{link}_{\Delta} \sigma, k\right)=0$ for $i<\operatorname{dim}\left(\operatorname{link}_{\Delta} \sigma\right)$. An equivalent condition is for the Stanley-Reisner ring of $\Delta$ to be a Cohen-Macaulay ring, and there is also an equivalent topological condition which makes no reference to the face structure of $\Delta$. Examples of complexes that are Cohen-Macaulay (over any $k$ ) include any triangulation of a sphere.

Every Cohen-Macaulay simplicial complex $\Delta$ is pure, i.e., every pair of facets have the same dimension. There is a related notion for general complexes. The pure d-skeleton of a simplicial complex $\Delta$ is the subcomplex generated by all faces of dimension $d$. We say that a complex is sequentially Cohen-Macaulay over $k$ if the pure $d$-skeleton is CohenMacaulay (over $k$ ) for all $d$. Once again, there are equivalent ring-theoretic and topological conditions for the sequentially Cohen-Macaulay property. Any pure sequentially CohenMacaulay complex is Cohen-Macaulay.

A simplicial complex $\Delta$ is shellable if there is an ordering $\sigma_{1}, \ldots, \sigma_{m}$ of the facets of $\Delta$ such that the intersection of $\sigma_{i}$ with the complex generated by $\sigma_{1}, \ldots, \sigma_{i-1}$ is pure $\left(\operatorname{dim} \sigma_{i}-1\right)$-dimensional. When possible, we avoid this definition and work through the condition of $k$-decomposability introduced in Section 3.

Any shellable complex is sequentially Cohen-Macaulay over any $k$, and we view shellability as a combinatorial condition for a complex to be sequentially Cohen-Macaulay. Since the results we prove will be independent of the field or ring $k$, we henceforth suppress $k$ from our notation.

A linear resolution of an ideal $I$ in a ring $R$ is a minimal free resolution of $R / I$ satisfying certain properties - the exact definition will not be important to us, as we work through the characterization of Eagon and Reiner [11] that the property of possessing a linear 
resolution is Alexander-dual to being Cohen-Macaulay. As the details are somewhat complicated, and only required in Section 6, we defer further discussion to that section.

\section{$3 \quad k$-decomposable complexes}

Provan and Billera [27] introduced a definition of $k$-decomposability for pure complexes. For $k=0$ these are known as vertex decomposable complexes, and the definition of vertex decomposable complexes was extended to non-pure complexes by Björner and Wachs [3]. We now give an analogous extension of $k$-decomposability to non-pure complexes for $k>0$.

The following definition was first made by Jonsson [22, Definition 2.10]:

Definition 3.1. Let $\Delta$ be a simplicial complex on vertex set $V$. Then a face $\sigma$ is called a shedding face if every face $\tau$ of $\operatorname{star}_{\Delta} \sigma$ satisfies the following exchange property: for every $v \in \sigma$ there is a $w \in V \backslash \tau$ such that $(\tau \cup\{w\}) \backslash\{v\}$ is a face of $\Delta$.

Remark 3.2. An equivalent condition to the exchange property of Definition 3.1 is the following: no facet of $\left(\operatorname{star}_{\Delta} \sigma\right) \backslash \sigma$ is a facet of $\Delta \backslash \sigma$.

Remark 3.3. In the case where $\sigma$ is a single vertex, the definition of shedding vertex specializes to that of Björner and Wachs [3, Section 11]. In the case that $\Delta$ is pure, the definition specializes to that of Provan and Billera [27, Definition 2.1].

Our main fact about shedding faces is the following generalization of [31, Lemma 6]:

Lemma 3.4. (Essentially Jonsson [22]) If $\sigma$ is a shedding face for a simplicial complex $\Delta$ such that both $\Delta \backslash \sigma$ and $\operatorname{link}_{\Delta} \sigma$ are shellable, then $\Delta$ is shellable.

Sketch. We first order the facets not containing $\sigma$ according to the shelling order of $\Delta \backslash \sigma$, followed by the facets containing $\sigma$ in the order indicated by the shelling of $\operatorname{link}_{\Delta} \sigma$. It is straightforward to verify this facet ordering is a shelling.

We see that the definition of shedding face can be viewed as a tool to build up a shelling of $\Delta$ by "sorting" the facets of $\Delta$.

Definition 3.5. A simplicial complex $\Delta$ is recursively defined to be $k$-decomposable if either $\Delta$ is a simplex or else has a shedding face $\sigma$ with $\operatorname{dim} \sigma \leq k$ such that both $\Delta \backslash \sigma$ and $\operatorname{link}_{\Delta} \sigma$ are $k$-decomposable. We consider the degenerate complexes \{\} and $\{\emptyset\}$ to be $k$-decomposable for all $k \geq-1$.

Definition 3.5 obviously extends the definition of vertex decomposability and pure $k$-decomposability.

Many of the theorems proved by Provan and Billera go through straightforwardly for our definition. Most interesting from the author's perspective is:

Theorem 3.6. (Jonsson [22]) A d-dimensional (not necessarily pure) simplicial complex $\Delta$ is shellable if and only if it is d-decomposable. 
Proof. Lemma 3.4 gives the $(\Leftarrow)$ direction.

Conversely, it follows directly from [2, Lemma 2.4] that the "minimal new face" contained in the last face of a shelling order is a shedding face. Induction then gives the $(\Rightarrow)$ direction.

Theorem 3.6 tells us that $k$-decomposability gives a hierarchical structure on the family of shellable complexes: every $k$-decomposable complex is also $(k+1)$-decomposable, and every shellable $d$-dimensional complex is $j$-decomposable for some $j \leq d$.

Some other theorems that extend easily are:

Proposition 3.7. If a simplicial complex $\Delta$ is $k$-decomposable, then for every face $\tau$ of $\Delta$ it holds that $\operatorname{link}_{\Delta} \tau$ is $k$-decomposable.

Proof. Entirely similar to [27, Proposition 2.3].

The following is stronger than [27, Proposition 2.4]:

Proposition 3.8. The simplicial complexes $\Delta_{1}$ and $\Delta_{2}$ are $k$-decomposable if and only if $\Delta_{1} * \Delta_{2}$ is $k$-decomposable.

Proof. The "only if" direction is entirely similar to [27, Proposition 2.4].

Conversely, suppose that $\sigma$ is a shedding face of $\Delta_{1} * \Delta_{2}$, with $\sigma_{i}=V\left(\Delta_{i}\right) \cap \sigma$. Let $\tau_{1}$ be any facet of $\Delta_{1}$ which contains $\sigma_{1}$, and $\tau_{2}$ similarly for $\Delta_{2}$ and $\sigma_{2}$. Then every face of the form $\rho_{1} \dot{\cup} \tau_{2}$ with $\sigma_{1} \subset \rho_{1}$ satisfies the shedding face exchange property, and (since $\tau_{2}$ is a facet) every face $\rho_{1}$ of $\Delta_{1}$ containing $\sigma_{1}$ satisfies the exchange property. Thus $\sigma_{1}$ and by symmetry $\sigma_{2}$ are shedding faces for $\Delta_{1}$ and $\Delta_{2}$. Since $\sigma_{1}$ and $\sigma_{2}$ are contained in $\sigma$, both have dimension $\leq k$, and at least one is non-empty.

Next, we notice that $\operatorname{link}_{\Delta} \sigma=\operatorname{link}_{\Delta_{1}} \sigma_{1} * \operatorname{link}_{\Delta_{2}} \sigma_{2}$, and by induction each of $\operatorname{link}_{\Delta_{1}} \sigma_{1}$ and $\operatorname{link}_{\Delta_{2}} \sigma_{2}$ is $k$-decomposable. (If $\sigma_{i}=\emptyset$, then we notice that $\operatorname{link}_{\Delta_{i}} \sigma_{i}=\Delta_{i}$.) Finally, $\Delta_{1} \backslash \sigma_{1}=\operatorname{link}_{\Delta \backslash \sigma} \tau_{2}$, which is $k$-decomposable by Proposition 3.7 .

Our main application of $k$-decomposability will come in Section 5, where we use it to prove that the independence complex of a chordal clutter is shellable.

Remark 3.9. Simon [28, Section 2.3] has introduced "clean ideal trees," an extension of $k$ decomposability via commutative algebra; however the concrete condition for a shedding face seems to better lend itself to constructing shellings.

We prove the following lemma for use in Section 6 .

Lemma 3.10. Let $\Delta$ be a vertex decomposable simplicial complex. Then the s-skeleton of $\Delta$ is vertex decomposable for any $s$.

Proof. Let $\Delta^{(s)}$ denote the $s$-skeleton of a simplicial complex. Clearly, $\operatorname{link}_{\Delta^{(s)}} v=$ $\left(\operatorname{link}_{\Delta} v\right)^{(s-1)}$, while $\Delta^{(s)} \backslash v=(\Delta \backslash v)^{(s)}$, so that by induction it suffices to produce a shedding vertex in $\Delta^{(s)}$. Then either $\Delta$ is a simplex, in which case every vertex of $\Delta^{(s)}$ is a shedding vertex; or else $\Delta$ has a shedding vertex, which is easily seen to remain a shedding vertex in $\Delta^{(s)}$. 
We close this section with a question, which we believe to be open even in the case of flag complexes $(m=2)$.

Question 3.11. What is the smallest $k$ such that if $\Delta$ is a shellable d-dimensional complex with $\mathcal{C}(\Delta)$ having maximum circuit cardinality $m$, then $\Delta$ is necessarily $k$-decomposable?

It is particularly natural to ask if every such complex is $(m-1)$-decomposable, as deleting an $(m-1)$-face preserves the maximum circuit cardinality condition.

\section{Chordal clutters}

Before introducing our definition of chordal clutters, we recall the definition and main structure theorem for chordal graphs. A graph is chordal if every induced cyclic subgraph of $G$ has length 3. A vertex $v$ of $G$ is simplicial if the neighborhood of $v$ in $G$ is a complete subgraph. The main theorem characterizing chordal graphs is:

Theorem 4.1. (essentially G. Dirac [8]) A graph $G$ is chordal if and only if every induced subgraph of $G$ has a simplicial vertex.

Most of the attempts in algebraic combinatorics at extending the definition of chordal to clutters have centered around extending the definition of simplicial vertex, and ours will be no exception.

Definition 4.2. Let $\mathcal{C}$ be a clutter. A vertex $v$ of $\mathcal{C}$ is simplicial if for every two circuits $e_{1}$ and $e_{2}$ of $\mathcal{C}$ that contain $v$, there is a third circuit $e_{3}$ such that $e_{3} \subseteq\left(e_{1} \cup e_{2}\right) \backslash\{v\}$.

In the case where $G$ is a graph, Definition 4.2 obviously agrees with the previous definition of a simplicial vertex.

Definition 4.3. A clutter $\mathcal{C}$ is chordal if every minor of $\mathcal{C}$ has a simplicial vertex.

Example 4.4. The following clutters are chordal:

1. Chordal graphs: If $G$ is a graph, then $G / v$ is (up to singleton circuits) the induced subgraph $G \backslash N[v]$. Hence the definition of chordal clutter specializes in graphs to the usual definition of chordal.

2. The complete $d$-uniform clutter $\mathcal{K}_{n}^{d}$ is the clutter with $n$ vertices and circuit set $\left(\begin{array}{l}V \\ d\end{array}\right)$. Since $\mathcal{K}_{n}^{d} / v \cong \mathcal{K}_{n-1}^{d-1}, \mathcal{K}_{n}^{d} \backslash v \cong \mathcal{K}_{n-1}^{d}$, and every vertex is simplicial, the complete $d$-uniform clutter is chordal.

3. Matroid circuits: Compare Definition 4.2 with Example 2.1. The simplicial vertex condition is exactly the weak circuit exchange property of matroids at a single vertex $v$. Thus every vertex of a matroid circuit clutter is simplicial. Since every deletion or contraction of a matroid gives another matroid, the circuit clutter of any matroid is chordal.

For example, $\mathcal{K}_{n}^{d}$ is the circuit clutter of a uniform matroid. 
Example 4.5. Van Tuyl and Villareal [30] define a clutter $\mathcal{C}$ to have the free vertex property if every minor of $\mathcal{C}$ has a free vertex, that is, a vertex appearing in exactly one circuit of $\mathcal{C}$. We observe that a free vertex is simplicial, so clutters with the free vertex property are chordal. Clutters with the free vertex property were shown to be shellable in [30, Theorem 5.3], and a restricted case of Proposition 5.2 for free vertices was shown in [25, Theorem 2.8].

Van Tuyl and Villarreal notice [30, Corollary 5.7] that if $G$ is a chordal graph, then the clutter $\mathcal{C}$ with vertices $V(G)$ and circuits consisting of all cliques in $G$ has the free vertex property. The Graham-Yu-Özsoyglu algorithm from database theory can be used to show that every clutter with the free vertex property has this form: a helpful reference is [1, especially Theorem 3.4]. Specifically, the Graham-Yu-Özsoyglu algorithm chooses a free vertex $v$ contained in a unique circuit $e$, and deletes $v$ if $e \backslash\{v\}$ is strictly contained in another circuit, and contracts $v$ otherwise - the algorithm terminates if and only if $\mathcal{C}$ is the clutter of cliques of a chordal graph.

Remark 4.6. Clutters (and more generally hypergraphs) which have the free vertex property have often been referred to as "acyclic". Since despite the name these clutters may have cycles, we prefer the free vertex property terminology.

Example 4.7. The clutter with circuits $\{1,2,3\},\{1,4,5\},\{2,3,4,5\},\{2,3,6\},\{4,5,6\}$ has simplicial vertex 1 , and is easily verified to be chordal; but is not a chordal graph or matroid circuit clutter, and does not have the free vertex property.

Example 4.8. Emtander [13], extending ideas from Hà and Van Tuyl [19], has a different but related definition of chordal for $d$-uniform clutters. Let a vertex $v$ be a completeneighborhood vertex if the induced subclutter on $S=\{x: x, v \in e\}$ is the complete $d$-uniform clutter, i.e. has circuits $\left(\begin{array}{l}S \\ d\end{array}\right)$. Emtander calls a $d$-uniform clutter "chordal" if every induced subclutter either has a complete-neighborhood vertex, or else no circuits.

A complete-neighborhood vertex is clearly simplicial in our sense, but Emtander requires only deletions to have simplicial vertices, while we require both deletions and contractions. Examples which are chordal in our sense but not in Emtander's are easy to come by (most matroids will do). An example which has complete-neighborhood vertices in every induced subclutter but is not chordal is the clutter $\mathcal{C}$ with circuits $\{1,2,3\},\{3,4,5\},\{5,6,7\},\{7,8,1\}$. Every induced subclutter of $\mathcal{C}$ has the free vertex property, but contracting $2,4,6$, and 8 leaves the cyclic graph $C_{4}$. (It follows immediately that $I(\mathcal{C})$ is not shellable or sequentially Cohen-Macaulay.)

\section{$5 \quad$ Shellability of the independence complex}

Our main goal of this section will be to prove Theorem 1.1 .

Recall [32, Lemma 6] that if $G$ is a graph with vertices $v$ and $w$ such that $N[v] \subseteq N[w]$, then $w$ is a shedding vertex in $I(G)$. Motivated by this result, we define a neighborhood containment pair of a clutter $\mathcal{C}$ to be a vertex $v$ and a circuit $e$ with $v \in e$ such that if $v \in e_{2}$ for any circuit $e_{2} \neq e$, then there exists an circuit $e_{3} \subseteq\left(e \cup e_{2}\right) \backslash v$. Thus, a simplicial vertex forms a neighborhood containment pair with any circuit containing it. 
Lemma 5.1. If $\mathcal{C}$ is a clutter with a neighborhood containment pair $(v, e)$ then $\sigma=e \backslash\{v\}$ is a shedding face of $I(\mathcal{C})$.

Proof. Suppose that $\tau$ is a face of $I(\mathcal{C})$ containing $\sigma$. Then $\tau \cup v$ contains $e$, so is not a face (and in particular $v \notin \tau)$. For $x \in \sigma$, if $(\tau \backslash x) \cup v$ is not a face of $I(\mathcal{C}$ ) (hence of $I(\mathcal{C}) \backslash \sigma)$, then $(\tau \backslash x) \cup v$ contains some circuit $e_{2}$ with $v \in e_{2}$. But then the neighborhood containment condition gives an $e_{3} \subseteq\left(e \cup e_{2}\right) \backslash v \subseteq \tau$, contradicting the choice of $\tau$ as a face. Hence any such $x$ can be exchanged for $v$, fulfilling the shedding face exchange axiom.

Proposition 5.2. If $\mathcal{C}$ is a clutter containing a simplicial vertex $v$, and if every proper contraction of $\mathcal{C}$ is shellable, then $\mathcal{C}$ is shellable.

Proof. Let $e_{1}, \ldots, e_{k}$ be the circuits containing $v$, and $\sigma_{1}, \ldots, \sigma_{k}$ be the associated shedding faces $e_{i} \backslash\{v\}$. Let $\mathcal{C}_{0}=\mathcal{C}$, and $\mathcal{C}_{i}$ be generated by the minimal sets of $\mathcal{C} \cup\left\{\sigma_{1}, \ldots, \sigma_{i}\right\}$, so that $I\left(\mathcal{C}_{i}\right)=I(\mathcal{C}) \backslash \sigma_{1} \backslash \cdots \backslash \sigma_{i}$.

Then since there is an $e^{\prime} \subseteq\left(e_{i} \cup e_{j}\right) \backslash v=\sigma_{i} \cup \sigma_{j}$, we get that $\mathcal{C}_{i-1}$ has some $e^{\prime \prime} \subseteq e^{\prime} \subseteq$ $\sigma_{i} \cup \sigma_{j}$. In $\mathcal{C}_{i-1} / \sigma_{i}$ the circuit $e^{\prime \prime}$ contracts to $e^{\prime \prime \prime} \subseteq \sigma_{j}$. In particular the minimal sets of $\mathcal{C}_{i-1} / \sigma_{i}$ are the same as those of $\mathcal{C} / \sigma_{i}$. We have shown that $\mathcal{C}_{i-1} / \sigma_{i}=\mathcal{C} / \sigma_{i}$.

It is straightforward to check that $v$ is simplicial in each of $\mathcal{C}_{1}, \ldots, \mathcal{C}_{k-1}$, and that the circuits of $\mathcal{C}_{i}$ containing $v$ are $e_{i+1}, \ldots, e_{k}$. By Lemma 5.1 we have that $\sigma_{i}$ is a shedding face in $I\left(\mathcal{C}_{i-1}\right)$. Every required link of the form $\operatorname{link}_{I\left(\mathcal{C}_{i-1}\right)} \sigma_{i}=I\left(\mathcal{C}_{i-1} / \sigma_{i}\right)=I\left(\mathcal{C} / \sigma_{i}\right)$ is shellable. The vertex $v$ is isolated in $I\left(\mathcal{C}_{k}\right)$, so that $I\left(\mathcal{C}_{k}\right)=I(\mathcal{C} / v) * v$ is shellable; while each $I\left(\mathcal{C}_{i}\right)$ is shellable by Lemma 3.4 and induction.

A short intuitive explanation of the proof of Proposition 5.2 is that the faces $\sigma_{i}=$ $e_{i} \backslash\{v\}$ are exactly the circuits added when $v$ is contracted, so that deleting all of the $\sigma_{i}$ 's from $I(\mathcal{C})$ leaves $(\mathcal{C} / v) \dot{\cup}\{v\}$ as the minimal non-face clutter.

Corollary 5.3. Let $\mathcal{C}$ be a clutter with maximum circuit cardinality $k$, such that every contraction of $\mathcal{C}$ has a simplicial vertex. Then $I(\mathcal{C})$ is $(k-2)$-decomposable, hence shellable and sequentially Cohen-Macaulay.

Proof. By induction and noting that each shedding face produced in Proposition 5.2 has dimension at most $k-2$.

We thus have the following specialization of Theorem 1.1:

Corollary 5.4. If $\mathcal{C}$ is a chordal clutter with maximum circuit cardinality $k$, then $I(\mathcal{C})$ is $(k-2)$-decomposable, hence shellable and sequentially Cohen-Macaulay.

We also break out the statement of Corollary 5.3 in the case where $\mathcal{C}$ is a graph.

Corollary 5.5. If $G$ is a graph such that $G \backslash N[A]$ has a simplicial vertex for any independent set $A$, then $G$ is vertex decomposable.

The family of graphs given in Corollary 5.5 is a considerably more general family than that of chordal graphs, including for example simplicial graphs [6], the family of graphs considered in [15, Theorem 3.2], etc. 


\section{Linear resolutions and Alexander duality}

Recall that the complement $\bar{G}$ of a graph $G$ is the graph with the same vertex set and with circuit set $\{x y: x y \notin E(G)\}$. We define the complement of a $d$-uniform clutter similarly for any $d$. An important theorem in the algebraic combinatorics of a chordal graph is:

Theorem 6.1. (Fröberg [17]) Let $G$ be a graph. Then the circuit ideal of $G$ has a linear resolution if and only if $\bar{G}$ is chordal.

In this section we generalize the "if" direction of Theorem 6.1 from chordal graphs to chordal clutters, in particular proving Theorem 1.2 . We will first need to recall some facts about Alexander duality.

\subsection{Review of Alexander duality}

The Alexander dual of a simplicial complex $\Delta$ (denoted $\Delta^{\vee}$ ) is the simplicial complex with vertices $V=V(\Delta)$ and facets $\{V \backslash e: e$ a circuit of $\mathcal{C}(\Delta)\}$. The vertex set that we consider $\Delta$ over has unusually great importance in this definition, and if we wish to emphasize the vertex set that we are operating over we will use a subscript, e.g. $\Delta_{V}^{\vee}$.

The Alexander dual allows us to reduce the question of the existence of a linear resolution to topological combinatorics:

Theorem 6.2. (Eagon and Reiner [11, Theorem 3]) Let $\Delta$ be a simplicial complex. The circuit ideal of $\Delta$ has a linear resolution if and only if $\Delta^{\vee}$ is Cohen-Macaulay.

In particular, one approach to proving the "if" direction of Theorem 6.1 is as follows:

Theorem 6.3. (Eagon and Reiner [11, Proposition 8]) If $G$ is a chordal graph, then $I(\bar{G})^{\vee}$ is vertex decomposable.

If $\Delta^{\vee}$ is shellable, then the circuit ideal is said to have linear quotients.

Remark 6.4. Theorem 6.2 tells us that classifying the circuit ideals with linear resolution is equivalent to classifying all Cohen-Macaulay complexes, which is likely intractable. Finding large classes of circuit ideals with linear resolutions remains an interesting problem.

We recall some standard facts about Alexander duality [7, 24]:

Lemma 6.5. If $\Delta$ is any simplicial complex on vertex set $V$ then

1. $\tilde{H}_{i}(\Delta) \cong \tilde{H}^{|V|-i-3}\left(\Delta^{\vee}\right)$.

2. $\left(\Delta^{\vee}\right)^{\vee}=\Delta$.

3. $(\Delta \backslash v)^{\vee}=\operatorname{link}_{\Delta} \vee v$ and $\left(\operatorname{link}_{\Delta} v\right)^{\vee}=\Delta^{\vee} \backslash v$.

4. $\Delta$ is pure of dimension $d$ if and only if $\mathcal{C}\left(\Delta^{\vee}\right)$ is $(|V|-d-1)$-uniform.

THE ELECTROnic JOURNAL OF COMBinAtorics 18 (2011), \#P208 
Remark 6.6. The Alexander dual has been studied in topological combinatorics at least as far back as [23, Section 6]. It has also been studied in the context of combinatorial optimization under the name blocker or transversal, and it is in this context that Lemma 6.5 parts (2) and (3) were first observed. We refer the reader to [7] for further background and references from the combinatorial optimization point of view, or to [24] from the algebraic combinatorics point of view.

\subsection{Alexander duals of complements to chordal clutters}

If $\mathcal{C}$ is a clutter, then define $c_{d}(\mathcal{C})$ to be the clutter with the same vertex set $V$ as $\mathcal{C}$ and circuit set $\{e \subseteq V:|e|=d$, e not a circuit of $\mathcal{C}\}$. In the special case that $\mathcal{C}$ is $d$-uniform, this is the complement of $\mathcal{C}$. We refer to the circuits of $c_{d}(\mathcal{C})$ as $d$-non-circuits of $\mathcal{C}$.

We start by relating contraction in $\mathcal{C}$ with contraction in $c_{d}(\mathcal{C})$ :

Lemma 6.7. Let $\mathcal{C}$ be a clutter with no circuits of cardinality $(d-1)$, and $v$ be a simplicial vertex. Then $c_{d}(\mathcal{C}) / v=c_{d-1}(\mathcal{C} / v)$.

Proof. Suppose by contradiction that $e$ is a $d$-non-circuit of $\mathcal{C}$ with $v \notin e$, and that $e$ is the only such non-circuit contained in the set $e \cup v$. Then the induced subclutter of $\mathcal{C}$ on the set $e \cup\{v\}$ is a complete clutter with one circuit removed $\left(\mathcal{K}_{d+1}^{d} \backslash\{e\}\right)$, which contradicts the hypothesis that $v$ is simplicial. It follows that every $d$-non-circuit of $\mathcal{C}$ contains a $(d-1)$-set $e^{\prime}$ which is a circuit of $c_{d}(\mathcal{C}) / v$, i.e. such that $e^{\prime} \cup\{v\}$ is a non-circuit of $\mathcal{C}$. Thus such $e^{\prime}$ are precisely the circuits of $c_{d}(\mathcal{C}) / v$.

Because there are no circuits with $d-1$ vertices in $\mathcal{C}$, the $(d-1)$-circuits of $\mathcal{C} / v$ are exactly the sets $e$ with $e \cup\{v\}$ a circuit of $\mathcal{C}$. We have that

$$
\{e:|e|=d-1, e \cup\{v\} \text { a non-circuit of } \mathcal{C}\}=c_{d-1}(\mathcal{C} / v)=c_{d}(\mathcal{C}) / v .
$$

Notice that $\mathcal{C} / v$ is in general not a uniform clutter, even if the starting clutter $\mathcal{C}$ was uniform. It is for this reason that we work with $c_{d}$, which is defined for every clutter, rather than with a more straightforward complement of $d$-uniform clutters.

Lemma 6.8. If $v$ is a simplicial vertex of a clutter $\mathcal{C}$ such that $\mathcal{C} \backslash v$ has at least one $d$-non-circuit $(d \geq 2)$, then $v$ is a shedding vertex in $I\left(c_{d}(\mathcal{C})\right)^{\vee}$.

Proof. Suppose that $\sigma$ is a facet of $\operatorname{link}_{I\left(c_{d}(\mathcal{C})\right)^{\vee}} v$, so that $\sigma=(V \backslash e) \backslash\{v\}$ for some $d$-noncircuit $e$ not containing $v$. (Such a facet exists by the condition requiring $\mathcal{C} \backslash v$ to have at least one $d$-non-circuit.) Since $d \geq 2$ there are vertices $w_{1}, w_{2} \in e$, and we let $e_{i}$ be the set $\left(e \backslash w_{i}\right) \cup\{v\}$.

If both $e_{1}$ and $e_{2}$ are circuits of $\mathcal{C}$, then $\left(e_{1} \cup e_{2}\right) \backslash v=e$ is also a circuit, a contradiction; so at least one $e_{i}$ is a $d$-non-circuit. But then $\tau=V \backslash e_{i}$ is a facet of $I\left(c_{d}(\mathcal{C})\right)^{\vee} \backslash\{v\}$ with $\tau=\sigma \cup\left\{w_{i}\right\}$, meeting the requirement for a shedding vertex.

We are now ready to prove:

Theorem 6.9. If $\mathcal{C}$ is a chordal clutter with minimum circuit cardinality $d$, then $I\left(c_{d}(\mathcal{C})\right)^{\vee}$ is vertex decomposable. 
Proof. We proceed by induction, with base cases as follows: If $c_{d}(\mathcal{C})$ has no circuits, then $I\left(c_{d}(\mathcal{C})\right)^{\vee}$ is the degenerate complex \{\} , which we defined to be vertex decomposable. If $d=1$ and there is a circuit in $c_{d}(\mathcal{C})$, then the facets of $I\left(c_{d}(\mathcal{C})\right)^{\vee}$ are some collection of codimension 1 faces of a simplex, hence vertex decomposable [11, proof of Proposition 8].

For $d>1$, let $v$ be a simplicial vertex of $\mathcal{C}$. Then

$$
\operatorname{link}_{I\left(c_{d}(\mathcal{C})\right)^{\vee} v}=I\left(c_{d}(\mathcal{C}) \backslash v\right)^{\vee}=I\left(c_{d}(\mathcal{C} \backslash v)\right)^{\vee}
$$

is vertex decomposable by induction, and

$$
I\left(c_{d}(\mathcal{C})\right)^{\vee} \backslash v=I\left(c_{d}(\mathcal{C}) / v\right)^{\vee}=I\left(c_{d-1}(\mathcal{C} / v)\right)^{\vee}
$$

is vertex decomposable by induction with Lemma 6.7 and minimality of $d$.

If $\mathcal{C} \backslash v$ has a $d$-non-circuit, then $v$ is a shedding vertex by Lemma 6.8 , hence $I\left(c_{d}(\mathcal{C})\right)^{\vee}$ is vertex decomposable. Otherwise, $v$ is contained in every circuit of $c_{d}(\mathcal{C})$, hence in no facet of $I\left(c_{d}(\mathcal{C})\right)^{\vee}$, so that $I\left(c_{d}(\mathcal{C})\right)^{\vee}=I\left(c_{d}(\mathcal{C})\right)^{\vee} \backslash v$, which is vertex decomposable by induction.

We have proved the following generalization of Theorem 1.2.

Corollary 6.10. If $\mathcal{C}$ is a chordal clutter with minimum circuit cardinality $d$, then the circuit ideal of $c_{d}(\mathcal{C})$ has linear quotients, hence a linear resolution.

As mentioned in Example 4.8, there are clutters such that every subclutter contains a complete-neighborhood vertex, but that are not chordal. We can however use a similar technique to show that clutters with a complete-neighborhood vertex in every induced subclutter are vertex decomposable, improving the previous result [14, Theorem 4.3] that such clutters are shellable:

Proposition 6.11. Let $\mathcal{C}$ be a d-uniform clutter such that every induced subclutter has a complete-neighborhood vertex. Then $I\left(c_{d}(\mathcal{C})\right)^{\vee}$ is vertex decomposable.

Proof. By induction we may assume that $\operatorname{link}_{I\left(c_{d}(\mathcal{C})\right)^{\vee} v} v I\left(c_{d}(\mathcal{C} \backslash v)\right)^{\vee}$ is shellable. A complete-neighborhood vertex $v$ is simplicial, thus either $v$ is a shedding vertex or else $I\left(c_{d}(\mathcal{C})\right)^{\vee}=I\left(c_{d}(\mathcal{C})\right)^{\vee} \backslash v$, exactly as in the proof of Theorem 6.9. It remains only to show that $I\left(c_{d}(\mathcal{C}) / v\right)^{\vee}$ is shellable.

Let $N=\bigcup_{v \in e}(e \backslash\{v\})$ be the neighborhood of $v$. The induced subclutter on $N$ is $\mathcal{K}_{|N|}^{d}$. By Lemma 6.7, $I\left(c_{d}(\mathcal{C}) / v\right)^{\vee}$ has circuits $\{e:|e|=d-1, e \cup\{v\}$ a non-circuit of $\mathcal{C}\}$, that is, all $e$ of cardinality $d-1$ such that $e \nsubseteq N$.

It follows that $I\left(c_{d}(\mathcal{C}) / v\right)^{\vee}$ is the pure $|V|-d-2$ skeleton of the complex $\Delta$ on $V \backslash\{v\}$ with the single non-face $V \backslash(\{v\} \cup N)$. The facets of $\Delta$ are a collection of codimension 1 faces of a simplex, hence $\Delta\left[11\right.$, proof of Proposition 8] and by Lemma $3.10 I\left(c_{d}(\mathcal{C}) / v\right)^{\vee}$ are vertex decomposable.

Corollary 6.12. (Emtander [13, Theorem 4.1]) Let $\mathcal{C}$ be a d-uniform clutter such that every induced subclutter has a complete-neighborhood vertex. Then the circuit ideal of $c_{d}(\mathcal{C})$ has linear quotients, hence a linear resolution. 
Our complement operation $c_{d}$ outputs a $d$-uniform clutter, even if the starting clutter was not uniform. If $\mathcal{C}$ is not $d$-uniform, then $I(\mathcal{C})^{\vee}$ is not pure, hence not Cohen-Macaulay; but it could still be sequentially Cohen-Macaulay. Algebraically, this corresponds with the circuit ideal being component-wise linear [21]. It might be interesting to find an extension of Theorem 6.9 involving non-uniform clutters and component-wise linear circuit ideals.

\section{$7 \quad$ Forbidden minors}

\subsection{Obstructions to shellability}

Wachs defined an obstruction to shellability to be a non-shellable simplicial complex such that every induced subcomplex is shellable. The obstructions to shellability that are flag complexes (independence complexes of graphs) were recently classified: Francisco and Van Tuyl [16] showed that chordal graphs are sequentially Cohen-Macaulay and that the $n$-cycle is an obstruction to shellability for $n \neq 3,5$. The author [32] showed that every complex containing no such cycle is shellable. We see a close relationship between the obstructions to shellability in flag complexes and the forbidden subgraphs of a chordal graph.

It is easier to study obstructions to shellability in the special case of flag complexes for at least two reasons. The first is that graphs are better studied than clutters, and so there were pre-existing theorems relating the forbidden subgraphs characterization of chordal graphs to the simplicial vertex characterization. The second is that every link in a flag complex can be expressed as an induced subgraph: $\operatorname{link}_{I(G)} v=I(G \backslash N[v])$. We try to partially remedy the latter with the following alternate definition:

Definition 7.1. A complex $\Delta$ is a dc-obstruction to shellability if $\Delta$ is non-shellable, but both every induced subcomplex and every link are shellable. Here $d c$ stands for "deletion-

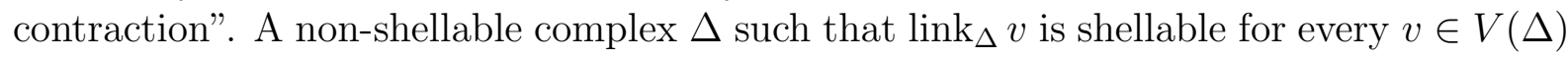
is a c-obstruction to shellability, and an obstruction to shellability in the sense of Wachs is a d-obstruction to shellability.

Example 7.2. The complex $\Delta$ with facets $\{\{1,2,3\},\{3,4,5\},\{1,5\}\}$ is a $d$-obstruction but not a $d c$-obstruction to shellability, since deleting any vertex leaves a connected complex with a single 2 -face, but $\operatorname{link}_{\Delta} 3$ is two disconnected edges, hence not shellable. Similarly for the family constructed in [31, Proposition 1].

Since $d$-obstructions to shellability allow the possibility of complexes where nonshellability is controlled by a proper (non-induced) subcomplex, we regard the definition of $d c$-obstructions to shellability as somewhat more natural. We comment that every pure c-obstruction to shellability is a Buchsbaum complex, and that conversely a Buchsbaum complex could be thought of as a pure "c-obstruction to Cohen-Macaulay."

Wachs conjectured [31] that there are a finite number of $k$-dimensional $d$-obstructions to shellability for any fixed $k$. Hachimori and Kashiwabara [20, Theorem 4.6] have recently shown that there are a finite number of $d$-obstructions in dimension $k$ if and only if 
there are a finite number of $d c$-obstructions in dimension $k$ - in particular, replacing " $d$-obstructions" with " $d c$-obstructions" in the conjecture of Wachs leaves an equivalent conjecture.

\subsection{Examples of forbidden minors}

A forbidden subclutter of some family $\mathcal{F}$ of clutters is a clutter $\mathcal{C}$ not in $\mathcal{F}$ such that every induced subclutter is in $\mathcal{F}$. A forbidden minor of some family $\mathcal{F}$ of clutters is a clutter $\mathcal{C}$ that is not in $\mathcal{F}$, but such that every minor (obtained by both deletion and contraction) is in $\mathcal{F}$.

Every nonshellable forbidden subclutter to chordality is a $d$-obstruction to shellability, while every nonshellable forbidden minor to chordality is a $d c$-obstruction to shellability (since these two families are the forbidden subclutters and forbidden minors to the family of clutters with every subclutter shellable). Thus, an approach to the obstructions to shellability problem is to understand the forbidden minors to chordal clutters.

For the case where $\mathcal{C}$ is a graph, we know that the forbidden minors to chordality are exactly $C_{n}$ for $n \geq 4$. The situation with general clutters is open, but it seems quite reasonable to ask whether every $d c$-obstruction to shellability is also a forbidden minor to chordality. We present several examples of infinite families of forbidden minors which are both. The hope is that a good understanding of these forbidden minors could lead to deeper understanding (or even a classification in the style of [32]) of obstructions to shellability.

Example 7.3. Let $\mathcal{Z}_{n}^{k}$ be the clutter with vertex set $\mathbb{Z}_{n}$ and circuits consisting of every $k$ consecutive elements. Thus, $\mathcal{Z}_{n}^{2} \cong C_{n}$, and more generally $\mathcal{Z}_{n}^{k}$ are the obvious $k$-uniform extension of the cyclic graphs. Any vertex (hence every vertex) of $\mathcal{Z}_{n}^{k}$ is simplicial if and only if $k=n$ or $n-1$, so $\mathcal{Z}_{n}^{k}$ is not chordal unless $k=n$ or $n-1$. Deleting any vertex leaves a clutter with the free vertex property, so $\mathcal{Z}_{n}^{k}(k \neq n, n-1)$ are forbidden subclutters to chordality. In some cases, for example $\mathcal{Z}_{5}^{3}$, they may also be forbidden minors.

We take a brief detour to discuss some cases when $\mathcal{Z}_{n}^{k}$ is not a forbidden minor to chordality, i.e., when $\mathcal{Z}_{n}^{k}$ has a non-chordal contraction.

Lemma 7.4. If $\ell k \leq n \leq \ell(k+1)$ and $k>2$, then $\mathcal{Z}_{n}^{k}$ has a contraction isomorphic to $\mathcal{Z}_{n-\ell}^{k-1}$.

Proof. The condition allows us to pick a set $S=\left\{v_{1}, \ldots, v_{\ell}\right\}$ vertices from $\mathbb{Z}_{n}=V\left(\mathcal{Z}_{n}^{k}\right)$ so that every 2 vertices in $S$ have $k$ or $k-1$ vertices between them. Contracting $S$ is easily seen to give $\mathcal{Z}_{n-\ell}^{k-1}$ as a minor.

E.g., $\mathcal{Z}_{6}^{3}$ is not a forbidden minor to chordality, since it contains a contraction minor isomorphic to the cyclic graph $C_{4}\left(=\mathcal{Z}_{4}^{2}\right)$.

More broadly, we could consider "clutters of cyclic type": clutters on vertex set $\mathbb{Z}_{n}$ with all circuits consisting of consecutive elements (possibly of different cardinalities). The next two examples, however, show that not all forbidden minors have this form; moreover, the results of Section 7.3 suggest that such a form is relatively uncommon. 
Example 7.5. Let $\mathcal{X}_{n}$ be the clutter with vertex set $[2 n]$ and circuits odd vertices\}, \{even vertices $\}$, and $\{i, i+1\}$ for all odd $i$. By symmetry no vertex is simplicial, and deleting or contracting any vertex leaves the same clutter (up to isomorphism). Any such deletion or contraction removes one of the two circuits with cardinality $n$, leaving a clutter with the free vertex property. Thus $\mathcal{X}_{n}$ is a forbidden minor to chordality for $n>1$.

The independence complex $I\left(\mathcal{X}_{n}\right)$ is the boundary complex of the $(n-1)$-dimensional cross-polytope with two opposing facets removed, a non-shellable complex. Hence $I\left(\mathcal{X}_{n}\right)$ is an $d c$-obstruction to shellability for $n>1$.

Example 7.6. Let $\mathcal{Y}_{n}$ be the clutter with vertex set $[2 n]$ and circuit set consisting of all $n$-sets except for $\{1, \ldots, n\}$ and $\{n+1, \ldots, 2 n\}$. It is straightforward to verify that every minor of $\mathcal{Y}_{n}$ is either a complete uniform clutter, or else a complete uniform clutter with one circuit removed. We notice that any vertex in the removed circuit of a latter such minor is simplicial, hence every proper minor of $\mathcal{Y}_{n}$ is chordal.

The independence complex $I\left(\mathcal{Y}_{n}\right)$ consists of all $(n-2)$ and lower dimensional faces, together with two disjoint $(n-1)$-faces. As the pure $(n-1)$-skeleton is disconnected, we have for $n>1$ that $I\left(\mathcal{Y}_{n}\right)$ is a $d c$-obstruction to shellability and $\mathcal{Y}_{n}$ is a forbidden minor to chordality.

We note that of the 2-dimensional $d c$-obstructions to shellability $M_{5}, M_{6}$, and $M_{7}$ that were considered by Wachs [31, Lemma 5], we have $M_{5} \cong I\left(\mathcal{Z}_{5}^{3}\right), M_{6} \cong I\left(\mathcal{X}_{3}\right)$, and $M_{7} \cong I\left(C_{7}\right)=I\left(\mathcal{Z}_{7}^{2}\right)$.

\subsection{Computational results}

Computation with GAP [18] yields exactly two forbidden minors to chordality on 5 vertices: the cyclic graph $C_{5}$ and the clutter $\mathcal{Z}_{5}^{3}$ discussed in Example 7.3. Both have homotopy type $S^{1} . I\left(\mathcal{Z}_{5}^{3}\right)$ is a $d c$-obstruction to shellability, while $I\left(C_{5}\right)$ is shellable.

On 6 vertices, a similar computation yields 294 (isomorphism classes of) forbidden minors to chordality on 6 vertices (out of 16,353 non-isomorphic 6 vertex clutters). There are an additional 96 clutters containing a $C_{5}$ minor (but no other non-chordal minor), all 96 of which are shellable. Of the 294 forbidden minors to chordality, 273 are shellable and 21 are not. The shellable forbidden minors to chordality on 6 vertices are too numerous to print here - a complete list and source code are available on my web page, currently at http://www. math. wustl.edu/ russw.

The 21 non-shellable forbidden minors to chordality are the $d c$-obstructions to shellability on 6 vertices. These clutters and their independence complexes are summarized in Table 1. The clutters and simplicial complexes are written in compact notation, so that, for example, 12 represents the set $\{1,2\}$. The fourth column of Table 1 represents the homotopy type of the pure top dimensional skeleton, as computed by automatic collapsing of free faces. Since each is a sphere of lower dimension than the top dimensional face, we see that none of these complexes are sequentially Cohen-Macaulay.

We notice that the first 16 rows of the table represent simplicial complexes consisting of two disjoint 2-faces, with enough edges between them to prevent a non-shellable minor. Line 1 represents the cylic graph $C_{6}$ and its independence complex, and Line 16 is 
Table 1: $d c$-obstructions to shellability on 6 vertices

\begin{tabular}{|c|c|c|c|}
\hline & Clutter of minimal non-faces & Independence complex & Top skel. \\
\hline 1. & $12,13,24,35,46,56$ & $145,16,236,25,34$ & $S^{0}$ \\
\hline 2. & $12,13,14,235,236,245,246,256,345,346,356,456$ & $156,234,25,26,35,36,45,46$ & $S^{0}$ \\
\hline 3. & $12,13,14,235,236,245,256,345,356,46$ & $156,234,25,26,35,36,45$ & $S^{0}$ \\
\hline 4. & $12,13,14,235,236,256,356,45,46$ & $156,234,25,26,35,36$ & $S^{0}$ \\
\hline 5. & $12,13,14,235,246,256,36,45$ & $156,234,25,26,35,46$ & $S^{0}$ \\
\hline 6. & $\begin{array}{l}12,13,145,146,235,236,245,246,256,345,346,356, \\
456\end{array}$ & $14,156,234,25,26,35,36,45,46$ & $S^{0}$ \\
\hline 7. & $12,13,145,146,235,245,246,256,345,36,456$ & $14,156,234,25,26,35,45,46$ & $S^{0}$ \\
\hline 8. & $12,13,145,146,245,26,346,35,456$ & $14,156,234,25,36,45,46$ & $S^{0}$ \\
\hline 9. & $12,13,145,234,236,245,246,345,346,56$ & $146,15,235,24,26,34,36,45$ & $S^{0}$ \\
\hline 10. & $12,13,145,236,24,345,346,56$ & $146,15,235,26,34,36,45$ & $S^{0}$ \\
\hline 11. & $12,13,145,24,345,36,56$ & $146,15,235,26,34,45$ & $S^{0}$ \\
\hline 12. & $12,13,234,235,245,345,46,56$ & $145,16,236,24,25,34,35$ & $S^{0}$ \\
\hline 13. & $\begin{array}{l}12,134,135,136,145,146,235,236,245,246,256 \\
\quad 345,346,356,456\end{array}$ & $\begin{array}{l}13,14,156,234,25,26,35,36,45 \\
\quad 46\end{array}$ & $S^{0}$ \\
\hline 14. & $12,134,135,136,145,234,236,245,246,345,346,56$ & $13,146,15,235,24,26,34,36,45$ & $S^{0}$ \\
\hline 15. & $12,134,135,146,235,246,256,36,45$ & $13,14,156,234,25,26,35,46$ & $S^{0}$ \\
\hline 16. & $\begin{array}{l}123,124,125,126,134,135,136,145,146,235,236 \\
\quad 245,246,256,345,346,356,456\end{array}$ & $\begin{array}{l}12,13,14,156,234,25,26,35,36 \\
\quad 45,46\end{array}$ & $S^{0}$ \\
\hline 17. & $12,134,135,146,235,246,256,345,346,356,456$ & $136,145,156,234,236,245,35,46$ & $S^{1}$ \\
\hline 18. & $12,134,135,234,246,345,346,56$ & $136,145,146,235,236,245,34$ & $S^{1}$ \\
\hline 19. & $12,134,256,35,46$ & $136,145,156,234,236,245$ & $S^{1}$ \\
\hline 20. & $\begin{array}{l}123,124,125,126,134,135,146,235,246,256,345 \\
\quad 346,356,456\end{array}$ & $\begin{array}{l}12,136,145,156,234,236,245,35 \\
\quad 46\end{array}$ & $S^{1}$ \\
\hline 21. & $1234,1235,1246,1356,2456,3456$ & $\begin{array}{l}1236,1245,1256,1345,1346,1456 \\
\quad 2345,2346,2356\end{array}$ & $S^{2}$ \\
\hline
\end{tabular}


isomorphic to the clutter $\mathcal{Y}_{3}$ discussed in Example 7.6. Lines 17 to 20 represent simplicial complexes consisting of an anulus formed by six 2-facets, together with some additional 1-dimensional facets. Line 19 is isomorphic to the clutter $\mathcal{X}_{3}$ discussed in Example 7.5. Line 21 is isomorphic to the clutter $\mathcal{Z}_{6}^{4}$ discussed in Example 7.3, via the ordering of vertices $1,2,4,6,5,3$. Lines 1 and 21 are the only clutters of cyclic type.

The computation took several hours on a $2.4 \mathrm{Ghz}$ MacBook, and involved enumerating over all 6 vertex clutters. The main technical difficulty was that computationally proving a complex to be non-shellable is very slow. However, checking for a simplicial vertex and checking for 4 or 5 vertex obstructions as minors are both fast, and give a short list of complexes to check for shellability. Indeed, as the non-shellable forbidden minors to chordality all had negative entries in their $h$-triangle, it was only necessary to find shellings for the shellable forbidden minors.

Remark 7.7. Hachimori and Kashiwabara [20] have more recently used non-computational methods to classify all 2-dimensional $d$-obstructions to shellability.

\section{References}

[1] Catriel Beeri, Ronald Fagin, David Maier, and Mihalis Yannakakis. On the desirability of acyclic database schemes. J. Assoc. Comput. Mach., 30(3):479-513, 1983.

[2] Anders Björner and Michelle L. Wachs. Shellable nonpure complexes and posets. I. Trans. Amer. Math. Soc., 348(4):1299-1327, 1996.

[3] Anders Björner and Michelle L. Wachs. Shellable nonpure complexes and posets. II. Trans. Amer. Math. Soc., 349(10):3945-3975, 1997.

[4] Anders Björner, Michelle L. Wachs, and Volkmar Welker. On sequentially CohenMacaulay complexes and posets. Israel J. Math., 169:295-316, 2009.

[5] Winfried Bruns and Jürgen Herzog. Cohen-Macaulay rings, volume 39 of Cambridge Studies in Advanced Mathematics. Cambridge University Press, Cambridge, 1993.

[6] Grant A. Cheston, E. O. Hare, S. T. Hedetniemi, and R. C. Laskar. Simplicial graphs. Congr. Numer., 67:105-113, 1988. Nineteenth Southeastern Conference on Combinatorics, Graph Theory, and Computing (Baton Rouge, LA, 1988).

[7] Gérard Cornuéjols. Combinatorial optimization, volume 74 of CBMS-NSF Regional Conference Series in Applied Mathematics. Society for Industrial and Applied Mathematics (SIAM), Philadelphia, PA, 2001. Packing and covering.

[8] G. A. Dirac. On rigid circuit graphs. Abh. Math. Sem. Univ. Hamburg, 25:71-76, 1961.

[9] Anton Dochtermann and Alexander Engström. Algebraic properties of edge ideals via combinatorial topology. Electron. J. Combin., 16(2):Research Paper 2, approx. 24 pp. (electronic), 2009.

[10] Art M. Duval. A common recursion for Laplacians of matroids and shifted simplicial complexes. Doc. Math., 10:583-618 (electronic), 2005. 
[11] John A. Eagon and Victor Reiner. Resolutions of Stanley-Reisner rings and Alexander duality. J. Pure Appl. Algebra, 130(3):265-275, 1998.

[12] Richard Ehrenborg and Gábor Hetyei. The topology of the independence complex. European J. Combin., 27(6):906-923, 2006.

[13] Eric Emtander. A class of hypergraphs that generalizes chordal graphs. Math. Scand., 106(1):50-66, 2010.

[14] Eric Emtander, Fatemeh Mohammadi, and Somayeh Moradi. Some algebraic properties of hypergraphs. Czechoslovak Math. J., 61(3):577-607, 2011.

[15] Christopher A. Francisco and Huy Tài Hà. Whiskers and sequentially CohenMacaulay graphs. J. Combin. Theory Ser. A, 115(2):304-316, 2008.

[16] Christopher A. Francisco and Adam Van Tuyl. Sequentially Cohen-Macaulay edge ideals. Proc. Amer. Math. Soc., 135(8):2327-2337 (electronic), 2007.

[17] Ralf Fröberg. On Stanley-Reisner rings. In Topics in algebra, Part 2 (Warsaw, 1988), volume 26 of Banach Center Publ., pages 57-70. PWN, Warsaw, 1990.

[18] The GAP Group. GAP - Groups, Algorithms, and Programming, Version 4.4.12, 2008.

[19] Huy Tài Hà and Adam Van Tuyl. Monomial ideals, edge ideals of hypergraphs, and their graded Betti numbers. J. Algebraic Combin., 27(2):215-245, 2008.

[20] Masahiro Hachimori and Kenji Kashiwabara. Obstructions to shellability, partitionability, and sequential Cohen-Macaulayness. J. Combin. Theory Ser. A, 118(5):16081623, 2011.

[21] Jürgen Herzog and Takayuki Hibi. Componentwise linear ideals. Nagoya Math. J., 153:141-153, 1999.

[22] Jakob Jonsson. Optimal decision trees on simplicial complexes. Electron. J. Combin., 12:Research Paper 3, 31 pp. (electronic), 2005.

[23] Gil Kalai. Enumeration of Q-acyclic simplicial complexes. Israel J. Math., 45(4):337$351,1983$.

[24] Ezra Miller and Bernd Sturmfels. Combinatorial commutative algebra, volume 227 of Graduate Texts in Mathematics. Springer-Verlag, New York, 2005.

[25] Susan Morey, Enrique Reyes, and Rafael H. Villarreal. Cohen-Macaulay, shellable and unmixed clutters with a perfect matching of König type. J. Pure Appl. Algebra, 212(7):1770-1786, 2008.

[26] James G. Oxley. Matroid theory. Oxford Science Publications. The Clarendon Press Oxford University Press, New York, 1992.

[27] J. Scott Provan and Louis J. Billera. Decompositions of simplicial complexes related to diameters of convex polyhedra. Math. Oper. Res., 5(4):576-594, 1980.

[28] Robert Samuel Simon. Combinatorial properties of "cleanness". J. Algebra, 167(2):361-388, 1994. 
[29] Richard P. Stanley. Combinatorics and commutative algebra, volume 41 of Progress in Mathematics. Birkhäuser Boston Inc., Boston, MA, second edition, 1996.

[30] Adam Van Tuyl and Rafael H. Villarreal. Shellable graphs and sequentially CohenMacaulay bipartite graphs. J. Combin. Theory Ser. A, 115(5):799-814, 2008.

[31] Michelle L. Wachs. Obstructions to shellability. Discrete Comput. Geom., 22(1):95103, 1999.

[32] Russ Woodroofe. Vertex decomposable graphs and obstructions to shellability. Proc. Amer. Math. Soc., 137(10):3235-3246, 2009. 